Catalogue and custom protein arrays

Alta Bioscience

BD Biosciences

CambridgePeptides

Clantech

EMDBiosciences

Epitome Biosystems

Eurogentec

Fluidigm

Hypromatrix

INTAYS

Irviltrogen

JPTPeptide Technologies

LC Sdiences

New England Peptide

PamGene

Panomics

Pepscan Systems

PeptideSpedaltylaboratories

Procognia

Protagen

ProteinOne

Protneteomix

QIAGEN

RayBlotech

Sigma-Aldrich

TeleChem International

Upstate (Millipore)

USBiomax

Whatman

Zeptosens

Zyomyx proteins
Custom peptide-array printing services

Cytometric bead arrays for multiplex protein analysis in solution, catalogueantibodies

Peptidesynthesis and purification services

Premade antbodymicroarrays

Human and murine cytokine an fbody arrays, various catalogueantibodies

Custorn-designed antibody ancyss

Custom protein, peptide and antibody arays, customand cata logueantibodies

BioMark micofluidicdynamic array immuncassay platiorm

AntbodyArays and Staining AntibodyAurays, catalogue antibodies

Custom peptide sets and arrays, custom polyclonal antibodies

ProtoArray human and yeast proteome chips, BioSource Luminex assays

Predesigned and custompeptidearrays for functional proteomics

Peptidearrays for phosphoproteomics epitopemapping

Custom peptide anays

PamChip flow-through peptide microarays for functionalstudies

Domain and transcriptionfactor arrays, an fbody and phosphoproteornic arays

PepChip peptidearrays for enzymeprofing epitopemapping

Specialized peptide sets for various applications

Specialized arrays for kinomics and glycomics

UNlchip protein arrays

Active Protein Aray platform for profing transcripfion factors, nuclear receptors and cancer

Protein and antibody fabicationand screening services

Qproteome GycoAuray kit Liquichip multiplex bead-based arrays

A variety of specialiced antibodyanays

Panoramaantibodyarrays, functional ancys and tissueextract arrays

Antbody arrays, microarraying instruments slides andsubstrates, reagents

BeadlytexMAP-based multiplex assays

Premade fluorescent or colorimetric an fbodycapturearrays

FAST Macro anfbody arrays, FAST slides and reagents, array services

Cely A reagents and equipment for revese-phase ancy preparation ancy services

Human and murine cytokine profling arrays
Birmingham, UK

San Jose, Callomia

Cambridge, UK

Mountain View, Callomia

Darmstadt Germany

Waltham, Massachusetts

Seraing, Belgium

South San Fran cisco, Calfomia

Worcester, Massachusetts

Cologne, Germany

Carlsbad, Calliomia

Berlin, Germany

Houston, Texas

Gadner, Massachusetts

's-Hertogenbosch, the Netherlands

Fremont, $\mathrm{Ca}$ alifornia

Lelystad, the Netherlands

Heidelberg Germany

Berkshire, UK

Dartmund Gemany

Bethesda, Maryland

Nantes, Fiance

Verlo, the Netherlands

Norcoss Georgia

St Louis Missourí

Sunnyvale, Calfornia

B lerica, Massachusetts

larnsvile, Maryland

Middesex, UK

Witterswil, Switzeland

Hayward Califomia wwwal tablosclencebhamac.

wmwbdblosdences.com

wuw.cambridgepeptides.com

wrwicloritech.com

www.emdblosclences.com

wwwrepitomeblosystems.com

uk.eur ogenteccom

wrw.fluidigncom

wuwhypromat rixcom

wwilntavis.com

wwwinvitrogencom

www.jptipom

wuwklcsciences.com

wuwnewenglandpeptide.com

www.pamgene.com

www.panomics.com

www.pepscannl

www.peptid.de

www.procognlacom

www.protagende

www.proteinone.com

www.protneteomb.com

wmwalagen_com

wwwrayblotech.com

wnw.sigmaal dich.com

www ar rayit.com

wumcupstatecom

wumblomaxus

www.whatman.com

wimixeptosens.com

www.xyomyxcom

Systems for array production

Arraylet

BiaAutomation

Bio-Rad Laboratories

Genetix

Genomic Solutions

LabNEXT

PerkinElmer

Scienion

VESS Sdentific
Inkjet-based microarray printers

MagnaSpotter for pin-based microarray printing

Arraying systerns, hybridization chambes, softwaretools

Pin-based arrayes and array scanners

OmniGrid microarrayers and hybrid zation chambers

Equipment for array printing and processing

Equipment for arrayproduction and scanning

sciFLEXARRAYER Plexo Dispenser for amay manufacture, specialized aray slide formats

Glass slide and compact discmicraarrayers
May feld, Scotland

Plana Texas

Hercules, Calfomia

NewMiton, UK

Amn Arbor, Michigan

Glerview, Iinois

Welleslex Massachusetts

Berlin, Germany

San Diego, Calfomia www ar rayjetcouk

wumblobautomationcom

wnwbib-radcom

wmwgenetbxcom

www.genomicsolutions.com

wumblatenextcom

las.perkinelmer.com

www.scienionde

wuw.vp-sdertific.com

\section{Array scanners}

Alphalmotech

Applied Precision

Biomedical Photometrics

Biomolex

BMGLABTECH

Fujifilm

LaVision BioTec

Mrailito

Molecular Devices

Tecan

VIDARSystems
Auray scanners

arrayWoRxbioch preades

Microarray scanners and analyfical software

Microarray rea ders based on isotopic detection

Microplatereaders for fuorescence and luminescence analysis

Scamers and analytical software

BiaAnalyzer CCD-based platiorms for microanayimaging

FMBIO fuorescent scanner

Microarray scanners and analyfical software

Microarray scanners and hybridization sta fions

Revolution 4200 microarray scamer
San Leandra. Callomia

lssaquah, Washington

Waterloo, Canada

Osla, Norway

Offenburg Germany

Tokya, lapan

Bielefield, Germany

Alameda Callfornia

Sunnyvale, California

Zurich, Switzeland

Herndon, Vrginia wwwal phainnotech.com

wrwwaplcom

wrwwconfocal.com

wumbibiomolex.com

wnwbibglabtech.com

wnw.fujifilmecom

wumclavisionblotecide

wrwwmiralilacom

wuwmolectil ardevices.com

wnwitecan.com

wwwumicroarrayscamer.com

\begin{tabular}{|c|c|}
\hline \multicolumn{2}{|c|}{ Ant ibodies and affinity reagents } \\
\hline 2lst Century Biochemicals & Custom antibody production and peptide synthesis \\
\hline Abcam & Catalogue antibodies and protein A-derived affibodies \\
\hline Abgent & Antbodies and antibody produdion \\
\hline Affibody & Afibodynon-immunoglobulin affinity molecules \\
\hline AnaSpec & Antbodies and customantibody production, peptide synthesis \\
\hline Antibodies Incorporated & Antbodies and antibody produdion \\
\hline BioDesign & Antbodies and manoclanal production \\
\hline BioGenes & Monocbonal and polyclonal antibodies, custom peptide synthesis \\
\hline Biolegend & Antbodies and fluorescent dyes \\
\hline Biomeda & Monoclonal and polyclonal antibodies, lectins \\
\hline EioTrend & Monocbonal and polyclonal antibodies, custom peptides \\
\hline
\end{tabular}

Marbora Massachusetts

Cambridge, UK

San Diego, Calforna

Bromma Sweden

San Jose, Callfornia

Davis, Calfomia

Saca, Maine

Berlin, Germany

San Diego, Calfomia

Foster City Callfornia

Cologne, Germany www.21stcenturyblacom

wnwabcamcom

wwwabgentcom

wmwatfibodycom

wmwanaspec.com

wwwantibodlesinc.com

wmwblodesign.com

wumblogeneside

wmwblidegend.com

wrumblomeda_com

wrwblotrend.com 


\begin{tabular}{|c|c|}
\hline COMPANY & PRODUCTS/ACTIVITY \\
\hline Cell Sciences & Monoclonal and polyclonal antibodies \\
\hline Cell Signa ling Technology & Monoclonal and poyclonal antibodies, screening kits and assays \\
\hline Chemicon (Millipore) & Custom andcatalogueanfbodies \\
\hline KPL & Catalogue and customantbodies, protein labelling reagents \\
\hline Lampire Biological Laboratories & Custom monoclonal and polyclonal antbody production \\
\hline Leinco Technologies & Catalogue and customantbodies \\
\hline Open Biosystems & Custom andcataloguepolyclonal and monoclonal antibodies \\
\hline QEDBioscience & Catalogue and customantbodies \\
\hline Rockland Immunochemicals & Antibodies and custom antibody production, detection reagents \\
\hline Santa Cruz Biotech & Catalogue polyclonal and monoclonal an fbodies \\
\hline Southern Biotech & Custom andcataloguemonoclonal and polyclonal antibodies \\
\hline Stratagene & Selected catalogue antibodies \\
\hline US Biological & Custom andcatalogueantbodies, lectins \\
\hline VWR & Antibodies from various suppliers, custom antibodies from $\mathrm{Neo}$ Clane \\
\hline
\end{tabular}

LOCATION

Canton, Massachusetts

Darvers, Massachusetts

Blerica, Massachusetts

Gaithersbugr, Maryland

Pipersilie, Penngivania

StLouis, Missouri

Huntsile, Alabama

San Diego, Calliomia

Gibertsvile, Pennsyhania

SantaCruz, Calfornia

Bimingham, Alabama

Lajolla California

Swampscott Massachusetts

West Chester, Pennsyivania
URL

wmw.cell sdences.com

mwu.cell signal.com

muw.chemicon.com

mwwikpl.

wmulampirecom

wwwlelnow.com

mww.openblosystems.0om

wuwsqedblacom

mwwurockdand-Inc.com

www.sebticom

www.southernbiotech.com

www.stratagene.com

mumustianet

mww.vwreom

\section{Slides and array substrates}

Accelir8

Erie Sdientific

Full Moon BioSystems

GE Healthare

GenTel BioSciences

Greiner Bio One

Microsurfaces

Nunc Brand

Ferce Biotechnology

Schott North America

Xenopore
OptAuraymicroarrayingslides

Glass slides and substrates for microarays

Protein microarray slides and semices

Codel ink activatedslides labelling reagents

Sides substrates and reagents for protein microarrays, array services

High-through put microarray plates

Surfacecoating for anayslides

Microanay sildecoatings

PATH protein microaray sides

Sides substrates and microarray reagents

Coated microariay slides, arrayerș array scanning sewices
Derver, Colorado

Portsmouth, New Hampshire

Sunnyale, California

Little Ohalfont, UK

Madison, Wisconsin

Monve, North Carolina

Minneapolis, Minnesota

Roskilde, Denmark

Rockford, Einois

Louisvile, Kentucky

Hawthome, Newlessey wrwwaced r.com

wuwserlemicroarray.com

www.fullmoonbio_com

wwwlamershambi osciences.com -

wwmgentelblacom

wwwgrelnerbiloonecom

www.proteinslides.com

wwwinunctr and.com

www.pler cenet.com

wwwus.schottcom/nexterion

wwwxenoporecom

\section{SPR and other platforms for label-free array analysis}

Biacore (GE Healthcare)

Bioforce Nanosciences

Bio-Rad Laboratories

Eco Chemie

Genoptics

GWC Technologies

IBIS Technologies

ICx Technologies

Lumera

NanoNord

Plasmonic

Protiveris

SRU Biesystems

XanTec
SPR instruments for high-throughput inteadionand kinetics analys's

Atomic-force-microscopy based platformfor nan oarray analysis

ProteOnXPR36SPR-based proteininteradionauray system, Bio-Plex suspension arrays

Autolab SPR instrumentation and accessories

SPRplatforms for perfoming ancy studes

SPR platforms suitable for protein microancy studies

SPRproducts and sensors

SensiQ SPR system for biomolecular in teraction analysis

Proteomic-Processor SPR instrument and array substrates

Cantion's cantilever technology for interaction detection

Products and instruments for SPR

Micro- and nanocanflever ancys for protein interaction analysis

BINDBiosensor technology for label-free interaction analysis

SPR biosensors, and array-compatible sildes
Uppsala,Sweden

Ames, lowa

Hercules, Callifornia

Utrecht theNefherlands

Orsay Cedex, France

Madison, Wisconsin

Hengela, the Netherlands

Washington DC

Bothel, Washington

Aalborg Denmark

Wallenfels, Germany

Rockvile, Maryland

Wobum, Massachusetts

Manster, Germany wumblacorecom

wwwbbloforcenano.com

wrubloilo-rad.com

wumsecochemien!

wum.genoptics-spr.com

www.guctechnologies.com

wwwlbls-spent

wuwlatecom

wumdumera_com

wwwnanonord.com

wuw.plasmoric de

wum.protheris com

wmwsrublesystemscom

wnwx antec.om

\section{Other array products}

Advalytix

Ariadne Genomics

Gyros

Imaging Research

Kreatech Biotechnology

Luminex

MesoScale Discovery

Promega

RocheApplied Science

Somalogic

SurModics

VigeneTech

- see advertisement
AurayBooster for improved hybridiation of protein arrays

Pathway Studio software for interpeefingmicroa aray data

Gyolab plationm for protein quantificationimmunoassays

AurayVision software for array analysis

ULS labeling technology foruse with protein arrays

xMAP microsphere platiorm for high-through put, solution-based mulfplex assays

Electrochemiluminescence-based platform for ancy analysis

HaloTag system for protein labeling

Reagents for protein labeling

Aptamer-based chips for protein de lectionand quantitation

Microarray stabiliner reagents

MicroVigenearray analysis software
Bumthal, Germany

Rockvile, Maryland

Uppsala,Sweden

Linton, UK

Amsterdam, the Netherlands

Austin Texas

Gaithersburg, Maryland

Madison, Wisconsin

Indianapols, Indiana

Boulder, Colorado

Eden Prairie, Minnesota

North Bilerica, Massachusetts wrwwadvalytix de

wumurarladnegenomics.com

wum.gyros.com

wwwilmagi ngresearch.com

wnwkkreatech.com

wwwduminexcorp.com

wuwmeso-scale com

wuw.promeg.com

wwwuroche-applied-sciencecom

www.somalogic.com

wunusurmodics.com

www.vigenetech.com 